\title{
Aperçu et actualités analytiques sur les armes chimiques
}

\section{Analytical outline and current events on chemical weapons}

Ivan RICORDEL

Laboratoire de Toxicologie de la Préfecture de Police, INPS, Paris

*Auteur à qui adresser la correspondance : Professeur Ivan RICORDEL, Laboratoire de Toxicologie de la Préfecture de Police, INPS, 2, Place Mazas, 75012 Paris - Tél : 0144754730 - Fax : 0144754725

Les principales données ont été exposées au XV $\mathrm{XV}^{\mathrm{em}}$ congrès de la SFTA, Paris, 6-8 juin 2007

(Reçu le 9 juillet 2007 ; accepté le 18 juillet 2007)

\section{RÉSUMÉ}

L'important stock d'armes chimiques dans le monde fait davantage craindre aujourd'hui son usage terroriste que le risque militaire de destruction massive.

Les toxiques terroristes candidats sont nombreux. Ceux qui font l'objet de recherches militaires constituent une gamme limitée principalement aux vésicants et organophosphorés. La dosimétrie biologique est l'élément princeps de la lutte. Elle concerne les produits en l'état, dégradés ou des adduits covalents sur les protéines (fixation sur la cystéine pour l'ypérite, cholinestérases phosphorylées pour les organophosphorés...) ou sur l'ADN. Les matrices concenées sont : les milieux environnementaux, les tissus agressés, le sang, l'urine, la salive, les organes de stockage. L'analyse, malgré son contexte d'emploi particulier; recourt aux mêmes moyens que ceux utilisés en toxicologie conventionnelle. La préparation de l'échantillon fait appel à l'extraction liquidelliquide, SPE, par fluide pressurisé ou en phase supercritique, et à la désorption thermique... Les outils sont la LC/MS et la CG/MS dans leurs divers modes : Scan, SIM, MRM, MS-MS et MS", MALDI TOF... La RMN est également utile.

Des perspectives thérapeutiques se font jour: De nouveaux inhibiteurs réversibles des cholinestérases (huperzine) pourraient potentiellement remplacer la pyridostigmine du traite-

\section{SUMMARY}

The important stock of chemical weapons in the world is more feared today for a terrorist use than a military risk of massive destruction. The terrorist poisons candidates are numerous. Those, which are the subject of military research, constitute a range limited mainly to the vesicants and organophosphorus compounds. Biological dosimetry is the main. element of the fight. It relates to the unchanged products, degraded products or covalent proteins adducts (cysteine adducts for yperite, cholinestérases phosphorylated for organophosphorus...) or ADN adducts. The matrixes concerned are : envirommental mediuns, attacked tissues, blood, urine, saliva, and intemal organs of storage. The analysis, in spite of its context of particular employment, resorts to the same means as those used in conventional toxicology. The preparation of the sample needs the liquid/liquid extraction, $S P E$, pressurized fluid or supercritical phase, and thermal desorption. The tools are LC/MS and GC/MS in their various modes: Scan, SIM, MRM, MS-MS and MSn, MALDI TOF... NMR is also useful. Therapeutic prospects energe. New reversible inhibitors of the cholinesterases (huperzine) could replace the pyridostigmine preventive treatment or better; the use of human or mutant cholinesterases or paraoxonase, which are also useful in decontamination. The emergency treatment could profit from "antiglutamatergic" neuropro- 
ment préventif ou mieux l'usage de cholinestérases ou paraoxonases humaines ou mutantes également utiles dans la décontamination. Le traitement d'urgence pourrait bénéficier de neuroprotecteurs « antiglutamatergiques ». La thérapie cellulaire constitue une approche prometteuse contre la neuropathologie des neurotoxiques organophosphorés. Enfin, la greffe cutanée de cellules souches et la dermoabrasion contrôlée devraient améliorer la réparation des lésions cutanées des vésicants.

\section{MOTS-CLÉS}

Ames chimiques, Ypérites, Organophosphorés, Lewisite, Adduits.

Le grand nombre de débats sur la crédibilité d'une menace chimique en tant qu'outil militaire de destruction massive témoigne de l'incertitude sur le sujet mais aussi de l'impact majeur dans l'inconscient collectif qu'a eu l'usage du chlore en 1915, du phosgène en 1916 et de l'ypérite en 1917 qui firent 100000 morts lors du premier conflit mondial.

En réalité, en dépit d'un arsenal mondial encore très conséquent qui devrait être complètement détruit aujourd'hui puisque les conventions internationales, depuis 1868 (Saint-Pétersbourg) ${ }^{1}$ puis 1874 (Bruxelles) ${ }^{2}$, en prévoyaient l'interdiction d'usage, on comprend que la prolifération ne se soit pas vraiment réduite avec la convention de Genève de 1925. En effet, si cette convention interdit « d'utiliser à la guerre, les gaz asphyxiants, les toxiques ou similaires et les moyens biologiques », elle n'interdit ni la fabrication, ni la détention de ces armes, ni la riposte... Toutefois, la situation devait être améliorée lors de la convention de Paris en janvier 1993 qui déterminait la disparition totale des stocks de munitions chimiques 10 ans après son début d'application, or sa mise en vigueur a eu lieu le 29 avril 1997 ...

182 états l'ont ratifié : $98 \%$ de la population mondiale, six autres pays l'ont signée sans la ratifier : Bahamas, Congo, Guinée-Bissau, Israël, Myanmar et République dominicaine. Seulement sept Etats ne l'ont ni signée ni ratifiée (Angola, Égypte, Iraq, Liban, République arabe syrienne, République populaire démocratique de Corée et Somalie).

Le devenir du stock non éliminé et la relative facilité de fabrication de ce type de produits " considérés longtemps comme le nucléaire du pauvre » ... font craindre davantage l'emploi terroriste que leur usage lors d'un

\footnotetext{
131 nations s'interdisent les « Armes dont I'emploi serait contraire aux lois de l'humanité »

216 nations s'interdisent « l'emploi du poison ou d'armes empoisonnées »
}

tectors. The cellular therapy constitutes a promising approach against the neuropathology of neurotoxic organophosphorus. Lastly, the cutaneous graft of stock cells and the controlled dermoabrasion should improve repair of the cutaneous lesions of the resicants.

\section{KEY-WORDS}

Chemical weapons, Yperites, Organophosphorus toxicants, adduct compounds.

conflit armé si l'on se réfère aux budgets des armées consacrés à leur défense.

Considérons aujourd'hui les seuls toxiques de guerre, les allégations et preuves de leur usage sont déjà légion. Parmi eux, citons :

- Le massacre de 2000 Kurdes à Halabja, par l'Irak, en 1988, qui serait imputable à des cocktails de vésicants et de neurotoxiques.

- ou encore l'attentat du 20 mars 1995 dans le métro de Tokyo avec l'usage de sarin, fort dilué, un neurotoxique organophosphoré, qui fit 12 morts, 700 hospitalisés dont 200 sauveteurs et des milliers de sujets désemparés (1).

Nous avons encore en mémoire la prise d'otages tchétchène, à Moscou, qui fit 120 morts et plus de 700 intoxiqués parmi les personnes présentes dans le théâtre Dubrowska, au cours de laquelle un anesthésique vétérinaire et vraisemblablement impur fut utilisé par les forces armées russes : le carfentanil, 10000 fois plus puissant que la morphine au plan pharmacologique. Il aurait par ailleurs (la preuve n'en a pas été apportée) été associé à un autre agent anesthésique volatil du type halothane ou isofurane. Ceci pourrait justifier, en dépit de l'index thérapeutique réputé correct du carfentanil, le caractère étonnamment délétère de l'intoxication et sa résistance à la naloxone (2). Il est vrai que le contexte d'emploi qui interdisait la maitrise de la diffusion, la forme galénique inhabituelle (aérosol), la capacité variable de stockage des graisses, le stress et le jeûne contrariaient pour le moins, l'analyse de la situation médicale. Il ne faut donc pas s'étonner dans un tel contexte que, 12 jours après l'assaut du théâtre, 67 otages et 9 sauveteurs aient été encore hospitalisés et pour certains dans un état grave.

Les armées concentrent quant à elles leurs efforts sur les toxiques les plus redoutables étant entendu que le riche arsenal potentiel du terrorisme concerne une toxicologie plus large qu'il convient d'étudier au cas par cas. Le contexte analytique qui nous intéresse davantage aujourd'hui est alors celui de la toxicologie conven- 
tionnelle qu'il faut adapter aux circonstances d'emploi. En ce qui concerne la stratégie de détection, les évolutions ont été très lentes mais depuis le début des années 2000, un certain frémissement dans la recherche est perceptible. Les fulgurants progrès des moyens dont nous disposons aujourd'hui associant méthodes séparatives (Chromatographie en phase gazeuse CG et Chromatographie en phase liquide haute performance HPLC, électrophorèse capillaire, nano chromatographie liquide nanoLC...) à la spectrométrie de masse (MS) dans toutes ses déclinaisons (single ion monitoring SIM, multiple reaction monitoring MRM, MS/MS, MS ${ }^{n}$, matrix-assisted laser desorption/ionization time of fligh MALDI/TOF...) augurent de progrès dont bénéficient aussi le domaine particulier des toxiques de guerre.

On parle aujourd'hui de dosimétrie biologique d'exposition aux agents $\mathrm{C}$. En amont, le produit en cause doit être détecté au plus vite pour déterminer les mesures de protection immédiates et adaptées à mettre en œuvre et définir le périmètre de protection des populations concernées.

Les moyens de détection utilisés pour ces missions font cohabiter archaïsme nécessaire et modernité dont la diffusion est encore limitée aujourd'hui (3). Des moyens assez frustres dotent encore les armées et la sécurité civile. Ils rendent nécessaire le contrôle de leur résultats pour confirmer ou lever les mesures de protection déclenchées. Il s'agit de papiers réactifs, de trousses de réactifs prêts à l'emploi, du Détindiv ${ }^{\mathrm{TM}}$ basé sur la réaction très sensible des organophosphorés sur l'acétyl cholinestérase dont ils inhibent le pouvoir de transformation d'un substrat coloré (limites de détection : $10 \mu \mathrm{g} / \mathrm{m}^{3}$ pour le sarin, $20 \mu \mathrm{g} / \mathrm{m}^{3}$ pour le soman, $50 \mu \mathrm{g} / \mathrm{m}^{3}$ pour le tabun $\left.20 \mu \mathrm{g} / \mathrm{m}^{3}\right)$. Les toxiques de guerre ne passent pas au travers de ce premier crible.

L'appareil AP2C (appareil portable de contrôle de la contamination chimique) est un photomètre de flamme sensible aux gaz riches en soufre (émission rouge) et phosphore à l'état gazeux (émission verte), muni d'une sonde de désorption électrothermique qui s'adapte aux toxiques liquides.

Les forces de secours sont également dotées de valises ergonomiques (LODITOX ${ }^{\mathrm{TM}}$ ) contenant des tubes Draeger $^{\mathrm{TM}}$ et des accessoires de prélèvement d'atmosphère. Le choix des tubes prédisposés dans le coffret est adapté aux groupes $\mathrm{CN}, \mathrm{F}$, S, thiodiglycol, As...

Les armées disposent d'un certain nombre de véhicules auto blindés (24). Ils comportent dans leurs accessoires en plus de l'AP4C (AP2C muni d'une sonde de désorption des toxiques liquides) et de la valise LODITOX ${ }^{\mathrm{TM}}$, d'un spectromètre de masse Brüker $^{\mathrm{TM}}$ et pour 12 d'entre eux, d'un chromatographe en phase gazeuse et de sa ligne de transfert au spectromètre. Ces moyens fonctionnent sur batteries.

Les spectromètres sont calibrés sur les masses caractéristiques des 200 principaux toxiques à « risque militaire » inclus dans des bibliothèques spécifiques OTAN qui comprennent également les toxiques industriels surveillés (TIC).

L'appareil est programmé pour analyser en continu les flux de désorption des gaz atmosphériques prélevés au moyen d'une canne/sonde électrothermique manipulée à la main de l'intérieur et qui transfère l'échantillon dans l'analyseur. Les échantillons de sol, liquides voire solides, sont prélevés au moyen d'une roulette siliconée, opérationnelle jusqu'à $60 \mathrm{~km} / \mathrm{h}$. Le prélèvement présenté à la sonde d'adsorption/désorption est analysable en introduction directe ou après séparation chromatographique.

La sécurité civile est également équipée depuis quelques années de laboratoires mobiles similaires (4). Le plus élaboré équipe les marins pompiers de Marseille. Il est muni d'un spectromètre de masse Brüker et de sondes de prélèvement. II fonctionne sur groupe électrogène remorqué par le camion-laboratoire. En dehors d'appareils de détection de la radioactivité, il possède également des tubes réactifs Draeger ${ }^{\mathrm{TM}}$, un explosimètre Oldham, l'AP4C ou un détecteur spectrométrique à mobilité ionique qui analyse en continu l'atmosphère aspirée à travers un filtre semi perméable s'affranchissant de l'oxygène. La source ionisante est l'Américium 241. Les ions sont séparés en fonction de leur masse et leur charge sous l'effet d'un champ électromagnétique. Les spectres de mélanges posent toutefois des problèmes d'interprétation. Les neurotoxiques de guerre, la léwisite, l'ypérite et l'acide cyanhydrique n'en poseraient pas.

Enfin un analyseur infra rouge à transformée de Fourrier analyse également en continu l'atmosphère aspirée et détecte tout composé organique volatil à plus de $100 \mathrm{ppb}$ dans l'atmosphère en 2 minutes.

L'équipement comprend aussi un CG/FID (détecteur à ionisation de flamme) utilisé simultanément pour les composés carbonés.

Des prélèvements de vapeurs sont réalisés sur tubes Tenax ${ }^{\mathrm{TM}}$ au moyen de pompes automatiques et des prélèvements de gaz sont faits au moyen de cannisters spécifiques.

L'analyse en TD (thermodesorption)/CG/MS prendrait 15 min selon les spécifications de la sécurité civile (30 000 produits sont référencés). Une fois la détection faite les systèmes portables évoqués préalablement permettent de zoner très rapidement l'espace dangereux 
par analyse de l'atmosphère.

L'unité de Marseille intervient une centaine de fois par an depuis 8 ans pour des alertes concernant dans $50 \%$ des cas des odeurs suspectes.

Il reste alors les infrastructures du territoire pour les autres moyens : spectrométrie d'absorption atomique électrothermique (SAAE), spectrométrie d'émission en plasma induit/spectrométrie d'émission atomique (ICP/AES) et ICP/MS voire la résonance magnétique nucléaire (RMN), auxquels s'ajoutent les techniques d'optoacoustique et d'interrogation neutronique non intrusives. Les dernières utilisent un rayonnement photonique issu de l'intéraction nucléaire entre la matière observée et un flux de neutrons thermiques (pour $\mathrm{Cl} / \mathrm{H} / \mathrm{N}$ ) ou rapides (pour $\mathrm{As} / \mathrm{OP}$ ) ou combinant les deux. Elles requièrent des détecteurs à scintillateurs à oxygermanate de bismuth ou à semi-conducteurs au Germanium de haute pureté. Leur interêt est l'accès à l'analyse de matrices difficiles à traiter comme le béton, le charbon, les ciments, les munitions chargées (5).

Les laboratoires experts spécialisés sont peu nombreux. Pour les armées, il s'agit du Centre d'étude du Bouchet et du CRSSA de Grenoble.

Les structures de la Police scientifique étudient la faisabilité d'une spécialisation dans ce domaine. Il y a là une niche de débouchés à venir.

Je m'intéresserai donc maintenant aux questions principalement d'ordre analytique touchant aux principaux toxiques de guerre notamment transposables au terrorisme dans les infrastructures « arrières 》. Quelles sont les particularités?

Les problèmes analytiques ne sont pas spécifiques. Le particularisme de ce domaine est la variété des matrices analysées (du prélèvement biologique aux peintures, polymères, matrices plastiques, parties métalliques, sols, végétaux etc.). Leur préparation doit particulièrement tenir compte de leur dangerosité potentielle. Il faut assurer la sécurité des opérateurs : rappelons qu'une goutte d'agent VX (ou A4 : [S-diisopropylaminoéthyl]-méthylthiophosphonate d'éthyle) sur la peau peut tuer un homme. Il faut par ailleurs assurer l'intégrité de l'échantillon en évitant tout risque de transfert du toxique à partir de ce prélèvement.

Des procédures recommandées dans le cadre de la convention de 1993 pour tous les échantillons environnementaux constituent une avancée dans ce domaine particulier.

Il n'est pas possible de développer ce thème aujourd'hui. Disons que la RMN du proton ou découplé du P, est un recours fréquent et qu'outre les techniques habituelles d'extraction liquide/liquide (LLE) et en phase solide (SPE), l'extraction en fluide pressurisé (ESP) (eau, acétone, méthanol, hexane à haute température maintenu liquide par haute pression) est fréquente de même que l'extraction en phase supercritique (EFS) qui présente une meilleure diffusion que l'ESP des solutés dans les solvants et des solvants dans la matrice. On utilise principalement le $\mathrm{CO}_{2}$ à $31{ }^{\circ} \mathrm{C}$ sous 72 bars pour les composés thermolabiles (5).

Considérons la dosimétrie biologique proprement dite. Elle est envisagée dans quatre situations principales : utilisation avérée ou simple allégation, opérations de démantèlement, attentats et inspections dans le cadre de la convention de 1993. Plusieurs objectifs peuvent être visés.

Le premier en réaction à l'événement est évidemment de confirmer la réalité de la menace donc d'identifier le produit en cause sur la zone d'emploi et sur les personnes impliquées voire dans les milieux biologiques des personnes blessées ou décédées.

Mais ces analyses peuvent être aussi nécessaires pour soutenir les études des mécanismes d'action et de distribution dans l'organisme ou de cinétique d'élimination. Elles le sont aussi pour optimiser les thérapeutiques, contrôler l'efficacité de la décontamination tant des personnes que des zones impliquées, définir la pertinence des marqueurs utilisables ou argumenter dans le cadre de contentieux...

Parmi les moyens d'analyse, l'observation des signes cliniques présentés par les sujets intoxiqués est primordiale (3-6). Les difficultés sont nombreuses comme à Tokyo et Moscou, en raison de l'ambiance, du stress, des fausses informations médiatisées ou parce que l'on a adjoint au toxique principal, des adultérants qui brouillent les pistes diagnostiques. A Tokyo comme à Moscou, il s'est passé plusieurs heures avant de confirmer l'identité du produit en cause. A Tokyo, le Sarin n'a été évoqué qu'à $\mathrm{T}+2$ heures 30 (1er résultat montrant l'effondrement des cholinestérases sanguines des intoxiqués). La confirmation du laboratoire expert n'a été donnée qu'à $\mathrm{T}+15$ heures mais la connaissance du résultat n'a pas pour autant modifié, dans certains hôpitaux, les traitements engagés contre les cyanures.

Les produits concernés sont principalement les moutardes au soufre (ypérite (HD dans la nomenclature OTAN)), les moutardes à l'azote (HN2 à HN3), les composés organophosphorés (tabun (GA), sarin (GB); soman (GD), cyclohexylsarin (GF), VX, VX-russe (R-33)).

$\mathrm{D}$ 'autres produits mériteraient un développement car ont été utilisés : un vésicant également toxique par l'arsenic qu'il contient, la léwisite (L), le cyanure d'hydrogène $(\mathrm{AC})$, le benzylate de quinuclidinol $(\mathrm{BZ})$, le phosgène (CG), les trichotécènes ( $\mathrm{T} 2$, désoxy-nivalénol...), la ricine, la toxine botulinique... Cette liste n'est 
qu'indicative et ne seront abordés ici que quelques exemples.

Les formes à analyser doivent être choisies selon les délais qui séparent l'exposition du prélèvement. Il est possible de détecter les produits en l'état pendant quelques heures. Les produits de dégradation sont accessibles en moyenne pendant quelques jours (produits d'hydrolyse, de condensation sur les fonctions S$\mathrm{H}$ et d'oxydation). Enfin, il est possible de recourir aux adduits covalents sur cibles biologiques notamment sur les protéines et l'ADN pendant quelques mois (7-8-9).

Les matrices potentielles sont les tissus, sièges initiaux de l'agression (peau, voies respiratoires), le système sanguin (sang total, plasma, sérum), l'urine et salive, les plus faciles à collecter, ou parfois les organes de stockage (cheveux, foie, graisses) en post-mortem.

Les outils les plus utilisés recourent à la détection par spectrométrie de masse notamment pour les petites molécules polaires. Le mode «Scan » convient aux fortes quantités de métabolites présents, les modes SIM, MRM, MS/MS et MS" au plus grand cas de figure où l'on est confronté à des traces ce qu'illustrent les quelques applications caractéristiques suivantes.

L'Ypérite est très peu représentée à l'état natif même dans les phlyctènes qu'elle provoque, hormis dans de rares cas pour lesquels le blessé est immédiatement pris en charge (explosions lors de déminage). Très rapidement l'hydrolyse et/ou l'oxydation du soufre conduisent au thiodiglycol (TDG) et aux formes sulfoxyde et sulfone du TDG. L'ypérite réagit par ailleurs avec le groupe S-H de la cystéine du glutathion.

Enfin l'ypérite se fixe via la formation d'ions épisulfonium, sur les groupements $\mathrm{S}-\mathrm{H}, \mathrm{O}-\mathrm{H}, \mathrm{NH}_{2}, \mathrm{CO}_{2} \mathrm{H}$ des protéines (l'hémoglobine, l'albumine...) ou sur la guanine des acides nucléiques (figure 1).

Les lésions toxiques de l'ypérite (molécule alkylante, radiominétique) sont des phlyctènes énormes, apparaissant en 4 à 8 heures et cicatrisant difficilement. L'ypérité est un brûlé immunodéprimé présentant des risques respiratoires infectieux sévères et une anémie thrombocytopénique et leucopénique pouvant être extrême (5-6).

On dose depuis longtemps l'ypérite en CG/MS (10). De même le thiodiglycol ne pose pas de problème d'analyse en CG/MS même si certains auteurs transforment le TDG en ypérite par hydrolyse chlorhydrique à $120^{\circ} \mathrm{C}$ pour revenir au cas précédent. On recourt à une déconjugaison par un mélange de sulfatases et glucuronidases puis après purification du dérivé en SPE $\mathrm{C} 18$, on dérive les esters penta fluoro benzoyles ou hepta fluoro butyryles en présence d'étalon interne deutéré (2H8TDG). R.M. Black et al. détectent en CG-
$\mathrm{NICI}$ (ionisation chimique en ions négatifs)-MS/MS, le fragment caractéristique $\mathrm{m} / \mathrm{z}=510$ (11).

Les mêmes auteurs injectent la forme majoritaire sulfoxyde (TDGO) après réduction préalable (l'oxygène du sulfoxyde pose problème pour la dérivation) par addition à l'urine de trichlorure de titane. Le reste est identique. La sensibilité est de $1 \mathrm{ng} / \mathrm{mL}$ (12).

Wils et al. déterminent des teneurs de 5 à $100 \mathrm{ng} / \mathrm{mL}$ (max 330) dans l'urine de sujets ypérités 5 à 10 jours après l'exposition et de 3 à $8 \mathrm{ng} / \mathrm{mL}, 8$ jours plus tard (7).

R. M. Black et al. analysent dans l'urine les produits d'addition $\mathrm{HD} /$ glutathion métabolisés par les $\beta$-lyases (figure 2) avec une sensibilité correcte de $0.1 \mathrm{ng} / \mathrm{mL}$ en CG/CI/MS SIM, soit après réduction puis purification en SPE sur $\mathrm{C} 8$ puis en CG-PICI (ionisation chimique en ions positifs)-MS/MS $\left(\mathrm{NH}_{3}\right)$ - ils détectent l'ion fragment moléculaire $\mathrm{m} / \mathrm{z} 232\left(\mathrm{M}+\mathrm{NH}_{4}{ }^{+}\right)$et l'ion fragment fils $\mathrm{m} / \mathrm{z} 75\left(\mathrm{MeSCH}_{2} \mathrm{CH}_{2}\right)^{+}(11)$-, soit directement en LC-ES (electrospray)-MS/MS à travers une colonne de polystyrène divinyl benzène (PSDVB) (7).

On peut aussi s'intéresser à l'analyse des produits métabolisés par des $\beta$-lyases puis conjugués sur la $\mathrm{N}$ acétyl cystéine en LC-ES-MS/MS en ions négatifs en triple quadripole avec une sensibilité de $1 \mathrm{ng} / \mathrm{mL}$ (7).

Filder et al. analysent les produits d'addition HD/ADN qui peuvent être détectés sur la peau, le sang, I'urine. Il s'agit principalement de l'adduit N7-(2-Hydroxy-éthyl thio éthyle)-guanine (figure 3) séparé en SPE C18 puis analysé en LC-ES-MS/MS par l'ion moléculaire $\mathrm{MH}^{+}=$ $\mathrm{m} / \mathrm{z} 256$ qui donne l'ion fils $\mathrm{m} / \mathrm{z} \quad 105$ $\left[\mathrm{CH}_{2} \mathrm{CH}_{2} \mathrm{SCH}_{2} \mathrm{CH}_{2} \mathrm{OH}\right]^{+}$(13). D. B. Ludlum et al. obtiennent par une méthode voisine, un seuil de sensibilité est de $8 \mathrm{pg}$ injecté $(0.2 \mathrm{ng} / \mathrm{mL})$ (14).

1 à $2 \%$ de l'ypérite se fixe spécifiquement sur la valine terminale de l'hémoglobine $\mathrm{Hb}$. Les auteurs clivent l'adduit de l'hémoglobine par une réaction d'Edmann modifiée au moyen du penta fluoro phényl isothiocyanate. Le résidu évaporé à sec redissous dans le toluène est dérivé avec l'anhydride hexafluorobutyrique. La limite de quantification est de $0.1 \mu \mathrm{mol}$ (après l'injection à des ouistitis d'une dose de $4.1 \mathrm{mg} / \mathrm{kg}$, les auteurs détectent l'adduit valine terminale pendant 94 jours en CG-NICI-MS (13).

L'adduit majoritaire est sur l'histidine. Il est stable après hydrolyse dans $\mathrm{HCl} 6 \mathrm{~N}$. R. M. Black et al. font une dérivation avec le chlorure de 9 fluorenylméthoxycarbonyle et détecte en LC-ESP-MS/MS (15-16). Price, en 2002, propose une méthode de contrôle de l'exposition à l'ypérite en MALDI TOF/MS sur l'hémoglobine adduite intacte. Il observe beaucoup d'espèces alkylées mais le recul n'est pas suffisant pour 
retenir la méthode en diagnostic (17).

Noort et al. ont mis en évidence en LC-ES-MS/MS que l'ypérite donne également des adduits sur l'unique résidu cystéine-34 libre de l'albumine (18). L'avantage de la méthode est la facile séparation de l'albumine par chromatographie d'affinité. Son inconvénient, par rapport à l'hémoglobine, est la durée plus courte de vie des adduits (20 à 25 jours contre 120). Cette technique est applicable aux moutardes à l'azote. La sensibilité est de $1 \mathrm{nM}$.

Enfin, Korte et al. proposent l'analyse des adduits de l'ypérite sur les résidus aspartiques et glutamiques des protéines sanguines. Ils séparent les protéines, hydrolysent les esters, extraient le thiodiglycol qu'ils dérivent et détectent en CG-NICI-MS. La sensibilité correspond à $2 \mathrm{nM}$ d'ypérite (19). Les auteurs suivent, chez le singe, l'élimination d'une dose intra veineuse de $1 \mathrm{mg} / \mathrm{kg}$ pendant 45 jours.

Cela a été appliqué à la kératine mais la manipulation est compliquée. Toutefois on a réalisé des anticorps contre la kératine sensibilisée à l'ypérite et un kit d'immunofluorescence a été conçu sur ce principe pour la détection de l'ypérite sur la peau (20).

Des applications sur les moutardes à l'azote ( $\mathrm{HN}$ dans la classification OTAN) sont voisines. Les produits d'hydrolyse des HN dans l'urine sont détectés en LCES-MS/MS directe (21). Comme pour l'ypérite au soufre, les produits d'addition HN-like sur l'albumine impliquent le résidu libre cystéine-34. D. Noort et al. opèrent en LC-ESP-MS/MS (18).

Le produit principal d'hydrolyse de la léwisite, dont l'action vésicante se double de l'action toxique de l'aisenic, est l'acide 2 chlorovinyl arsenieux (CVAA : figure 4). Jakubowski et al. dosent l'arsenic, dans l'urine ou le sang, après séparation chromatographique en absorption atomique, AES et EI (impact électronique)/MS en SIM (1999). La limite de quantification est voisine de $100 \mathrm{ng} / \mathrm{mL}$ (22). Fidder et al. dérivent une première fois avec le propre antidote de la léwisite, un dithiol (le dimercaptopropanol ou BAL, British-Anti-Lewisite), qui en même temps déplace les résidus de vésicant liés à la cystéine 93 et 112 de l'hémoglobine. Le complexe est extrait en SPE C18 et dérivé une seconde fois par l'hepta fluoro butyryle imidazole sur l'hydroxyle restant libre sur le dithiol. L'analyse se termine par CGEI-MS en SIM ( $\mathrm{m} / \mathrm{z}$ qualifiant 454). La limite de quantification est de $20 \mathrm{pg}$ injecté (23).

La méthode la plus sensible est celle de J. V. Wooten et al. (24). Ils proposent une variante en dérivant avec le 1, 3 propane dithiol directement sur l'urine en tampon acétate et en pratiquant une microextraction en phase solide (SPME) in-situ sur fibre de polydiméthylsiloxa- ne (PDMS) et en terminant en CG-EI-MS. La limite de quantification obtenue est de $7.4 \mathrm{pg} / \mathrm{mL}$ déterminée sur l'ion moléculaire.

Les neurotoxiques organophosphorés (OP) sont les plus étudiés (figure 5). Les lésions toxiques de ces composés sont plus familières puisque proches de celles des pesticides organophosphorés. Pour mémoire, par leur action cholinergique sur les récepteurs muscariniques, ils entraînent des effets sur les glandes, les muscles lisses et les nerfs crâniens, par leur stimulation des récepteurs nicotiniques, ils sont responsables des effets sur les muscles du squelette, sur les synapses ganglionnaires et sur les surrénales. Cela se traduit selon le produit en cause, la dose et la voie d'administration par une gradation plus ou moins homogène des symptômes suivants (6) : salivation, nausées, vomissements, oppression thoracique, bronchorrhée, dyspnée, sudation, pâleur, cyanose, bradycardie inconstante ou tachycardie, larmoiement, myosis, mictions involontaires, faiblesse musculaire, tremblements, fasciculations, secousses musculaires, paralysie flasque, inconscience, convulsions et défaillance respiratoire. La mort peut intervenir en quelques minutes. Le soman est plus neurotoxique et à dose importante, la paralysie respiratoire est aggravée de l'encombrement des voies aériennes par des sécrétions profuses et de plus, les convulsions dominent en quelques minutes avec installation de lésions cérébrales irréversibles.

Le métabolisme des organophosphorés donne des acides méthylphosphoniques alkylés présents dans le sang et l'urine. Les acides desalkylés ne sont présents qu'à l'état de traces (figure 6). Le dérivé correspondant au VX (EMPA : éthyl méthyl phosphonate) subit en plus, une S méthylation rapide dont le produit (méthyl thio éthyl diiso propylamine) est facilement extractible et identifiable par simple CG-MS (figure 7). Par ailleurs comme les ypérites, ces composés font avec les protéines (en particulier avec les cholinestérases) et avec l'ADN, des adduits détectables plusieurs semaines après l'exposition.

Rappelons que la méthode colorimétrique d'Ellman qui mesure la réduction de l'activité cholinestérasique a été longtemps la seule approche biologique possible de l'intoxication. Elle est encore fort utile mais elle n'identifie pas l'OP, n'est pas spécifique, ne signifie rien pour une chute de $20 \%$ et pour les trìs faibles valeurs et enfin n'est pas correctement corrélée à la gravité relative.

Les produits non métabolisés ne sont présents que pour des sangs collectés très tôt après l'exposition. De nombreuses méthodes existent pour l'expérimentation animale en CG/MS et CG/NPD (détecteur azote phosphore) applicables aux acides méthylphosphoniques alky- 
lés et à l'acide méthylphosphonique. Les produits d'hydrolyse des acides alkylméthylphosphoniques (RMPAc) font aussi l'objet de nombreux protocoles fondés en général sur une extraction SPE (C18, anionique, polymère), la préparation de dérivés pentafluorobenzylés avec le bromure de penta fluoro benzyle en milieu $\mathrm{K}_{2} \mathrm{CO}_{3}$ dans l'acétonitrile maintenus à $90^{\circ} \mathrm{C}$ pendant 1 heure puis lavés sur fluorisil et analysés en CG-NICI-MS ou MS/MS. J. R. Ritches et al. ont mis au point cette méthode, non publiée, pour le sarin (7), le soman et le cyclosarin. S.-A. Fredriksson et al. l'ont publiée avec en plus une application au VX (25). Cette méthode extrêmement sensible mise en œuvre sur un spectromètre magnétique hybride donne une sensibilité de quelques attogrammes sur des standards et de moins de $0.1 \mathrm{ng} / \mathrm{mL}$ sur des matrices biologiques. Ces mêmes auteurs obtiennent un seuil de quantification de 0.5 à 1 ng sur un système analyseur à piégeage d'ions (ITD : Ion Trap Detector). L'EMPA provenant du VX est 10 fois moins sensible.

Miki et al. en 1999 font une dérivation au moyen d'un catalyseur de transfert (tri n-butyl-méthylphosphonium) lié à un polymère. La méthode est compliquée mais ils obtiennent une limite de quantification (LOQ) de $0.06 \mathrm{ng} / \mathrm{mL}$ en CG/NICI/MS (26). Les autres dérivations (méthylation et silylation) sont moins bonnes.

La LC est généralement moins sensible que la CG/MS pour les acides alkyl méthyl phosphoniques toutefois Noort et al. publient une méthode correcte en micro LC/ES/MS-MS pour le soman. Ils mettent en évidence le 2 - isopropyl méthyl phosphonate dans le sérum de patients. Ils réalisent d'abord une extraction classique LLE (isobutanol/toluène 1/1) puis séparent sur colonne HPLC échangeuse d'anions (microbore PRPX100). La phase mobile est le mélange solvant : acétonitrile 1, eau 1 , acide formique $0.5 \%$. La limite de quantification obtenue est de 2 à $4 \mathrm{ng} / \mathrm{mL}$ (27).

Mais les produits initiaux et d'hydrolyse sont rapidement éliminés de l'organisme. Il faut alors avoir recours aux produits d'addition soit en les isolant de leurs combinaisons soit au sein de celles-ci. M. Polhuij et al. mesurent l'importance de l'inhibition de la butyryl cholinestérase par réactivation par les fluorures de l'enzyme inactive (figure 8) qui produit un phosphofluoridate qu'il analyse en CG/MS après extraction (28). Malheureusement cette technique ne s'applique pas à tous les OP notamment à ceux qui entraînent le phénomène d'« Aging » des enzymes (soman, tabun) qui interdisent leur réactivation.

Cette technique a été utilisée avec succès pour le sarin à Tokyo. D'autres se confronteront à ce même principe comme Jakubowski (29).
Une méthode compliquée proposée par Nagao et al. permet de s'affranchir du déplacement par les fluorures $\mathrm{F}$ - Le complexe acétylcholinestérase/sarin de la membrane de hématies est solubilisé par incubation avec de la trypsine, à $37^{\circ} \mathrm{C}$, pendant 24 heures. L'acide isopropyl méthyl phosphonique est traité par une phosphatase alcaline durant 48 heures. Les molécules résiduelles de grandes tailles sont ultrafiltrées. La solution aqueuse est évaporée à sec puis chromatographiée en CG/MS (30).

Doorn et al. (31) détectent en MALDI/TOF le peptide de l'enzyme inhibé par le malathion à partir d'une digestion trypsique de butyrylcholinestérase. Cette technique sera prise comme modèle par Jinchun Sun (32).

Les OP inhibent la butyrylcholinestérase par formation d'un pont covalent avec la sérine 198. Le peptide de 9 ou 14 acides aminés qui la contient est isolé par digestion avec de la pepsine ou la trypsine selon les auteurs. L'isolement est réalisé par chromatographie d'affinité sur gel de polyacrylamide (33). L'analyse peut se faire en LC/ES/MS-MS (34).

En ES/MALDI/TOF (32) (35), la solution matricielle est de l'acide alpha cyano 4 hydroxy-cinnamique (CHCA) dans l'acétonitrile à $10 \mathrm{mg} / \mathrm{L}$ mélangée à égales proportions avec une solution d'acide trifluoroacétique (7) (TFA à $0.1 \%$ dans l'eau [48] /acétonitrile [52]). On injecte $1 \mu \mathrm{L}$ de l'analyte à doser dans $1 \mu \mathrm{L}$ de matrice et $1 \mu \mathrm{L}$ de citrate d'ammonium mM. Le témoin interne est un mélange angiotensine II/vasopressine. On opère 300 shuts Laser. Les OP restent fixés pendant 5 à 16 jours. Les ions caractéristiques sont par exemple : $\mathrm{MH}^{+}=3008.6$ (digestion trypsine/soman), $\mathrm{MH}^{+}=876.3$ (digestion pepsine/soman), $\mathrm{MH}^{+}=916.4$ (digestion pepsine/sarin).

Bin Li et al. ont mis au point en MALDI/TOF, une technique séduisante qui permet d'une part, d'individualiser certains peptides de l'albumine liant le sarin sur la tyrosine 411 notamment mais aussi le diisopropy] fluorophosphonate (DFP), le chlorpyriphos-oxon, le dichlorvos, et d'autre part, de quantifier des teneurs de l'ordre de $0.07 \mathrm{pmol}$ d'adduit peptidique (36). L'intérêt est la concentration disponible d'albumine comparativement à celle des cholinestérases (facteur 10000). Des essais avec les carbamates sont en cours. La matrice retenue est l'acide di-hydroxy-2,5-benzoïque mais le CHCA est également utilisable. La méthode peut aussi être appliquée en triple quadripôle (37).

Un des buts d'une analyse fine des toxiques de guerre est l'amélioration de la protection des victimes mais aussi des personnels de secours impliqués. Quelles perspectives se dessinent sur ce plan? 
L'Armée disposait pour injection d'urgence aux premiers signes d'intoxication organophosphorée, d'une seringue autoinjectante à trois compartiments renfermant de l'atropine, de la pralidoxime et du diazépam (3). L'avisafone, précurseur soluble du diazépam a permis la réalisation de nouvelles seringues à 2 compartiments ; l'un contenant le lyophilisat des trois produits, l'autre l'eau pour préparation injectable qui dissout les médicaments au moment de l'injection et qui permet des durées de stockage plus longues en vue de pré positionner ces moyens en cas de catastrophe chimique (38). L'Armée dispose par ailleurs de syrettes d'atropine pour le traitement d'urgence et d'un prétraitement à la pyridostigmine susceptible de faciliter la réactivation des cholinestérases (3).

Il est envisagé d'améliorer l'efficacité de la seringue en substituant à la pralidoxime, un autre réactivateur de cholinestérase, le HI6 (1-[(4-aminocarbonyl-pyridinio]-méthoxy)-méthyl]-2-[hydroxyiminométhyl]-pyridinium) (39)(40) et de remplacer l'avisafone par le midazolam. Des projets avec le mélange kétamine/midazolam sont à l'étude (41-42).

A coté de l'atropine, des composés des anticholinergiques pourraient être employés. La gacyclidine (GK11) fut un des premiers étudiés par Lallement. Les résultats étaient prometteurs chez le primate (innocuité de 8 doses létales 50 de soman malgré un traitement tardif 45 minutes après le début des crises convulsives). Ont également donné des résultats, le MK801 (dizocilpine) et le TCP (dérivé tiénylé de la phencyclidine) (43).

Dans le domaine du prétraitement de l'intoxication organophosphorée, la pyridostigmine réduit la toxicité du soman mais est sans action sur le VX. Elle ne passe pas la barrière hémoméningée. D'autres molécules candidates ont été évoquées : physostigmine, ambémonium, aminostigmine, galanthamine (alcaloïde du perce neige du Caucase : Galanthus woronii). Dans ce domaine, on peut aussi parler de frémissement avec l'apparition d'inhibiteurs réversibles des cholinestérases traversant la barrière hémoméningée. Guy Lallement du CRSSA a étudié l'huperzine, alcaloïde extrait d'une mousse chinoise, avec laquelle il est parvenu à $100 \%$ de protection chez le cobaye contre la crise épileptique du soman avec de plus $100 \%$ de survie dans des conditions où la pyridostigmine ne protège pas de la crise et tue 6 animaux sur 7. L'huperzine se lie dans le cerveau au canal ionique associé aux récepteurs NMDA (N-méthyl-D-aspartate) de l'hippocampe en empêchant la crise convulsive (elle n'a pas d'action sur les récepteurs AMPA (acide alpha-amino3 hydroxy-5 méthyl-4isoxazole-propionique) et GABA (acide gamma anino butyrique)). Toutefois ces résultats encourageants doivent être confirmés et des études de toxicité approfondies doivent compléter ces travaux (44). La prophylaxie de ces toxiques pourrait être également grandement améliorée par l'hydrolyse des OP avant qu'ils n'atteignent leur cible d'où l'idée de réaliser des hydrolases mutantes génétiquement modifiées d'origine bactérienne, des butyryl et acétyl cholinestérase ou des paraoxonases humaines (45)(46).

Des phosphotriestérases (PTE) ont été incorporées dans des liposomes ou des nanoparticules pouvant libérer in vivo les hydrolases. Dans ce but des prolidases ont été extraites de mouches résistantes aux organophosphorés, certaines métalloenzymes et des enzymes désalkylantes (glutathion $S$ transférases) ont été envisagées (47).

À une autre échelle d'emploi, ces mêmes molécules, PTE, PON/HPBP (paraoxonases/human phosphate binding protein) (48) avec les cyclodextrines (49), permettraient de valider des dispositifs nouveaux de protection et de décontamination externe des personnes et du matériel. Il n'est pas exclu qu'elles puissent entrer dans la composition de topiques protecteurs cutanés ou de solutions décontaminantes en remplacement du gant poudreur utilisé par les militaires en cas de contamination.

Enfin l'axe analytique des militaires est celui de l'analyse évoquée ci-dessus, des adduits d'OP avec l'hémoglobine, les protéines, les cholinestérases et l'ADN.

Le traitement des lésions de l'ypérite pourrait bénéficier de la technique de dermoabrasion qui permet d'ajouter des molécules d'adressage accélérant la recommunication des cellules entre elles et constituant une matrice de régénération (50).

Les lésions neurotoxiques pourraient bénéficier prochainement de techniques de neurogenèse par greffe de cellules souches médullaires et neuronales. Cette thérapie génique appliquée à la neurogénèse implique des travaux de vérification de la survie de cellules souches endogènes et de leur mobilisation par cytokine de leur action neuroprotectrice anti-inflammatoire (51).

\section{En conclusion}

Je remercie les organisateurs de ce congrès d'avoir donné une tribune à ce domaine encore mal connu dont le mystère s'éclaircit en même temps que le danger s'éloigne du domaine strict du champ de bataille pour concerner maintenant une large population civile au travers des menaces d'attentats chimiques.

Conjoncturelle sans doute aujourd'hui, cette ouverture préfigure l'élargissement probable de vos futures préoccupations toxicologiques. 
Annales de Toxicologie Analytique, vol. XIX, n² 2, 2007

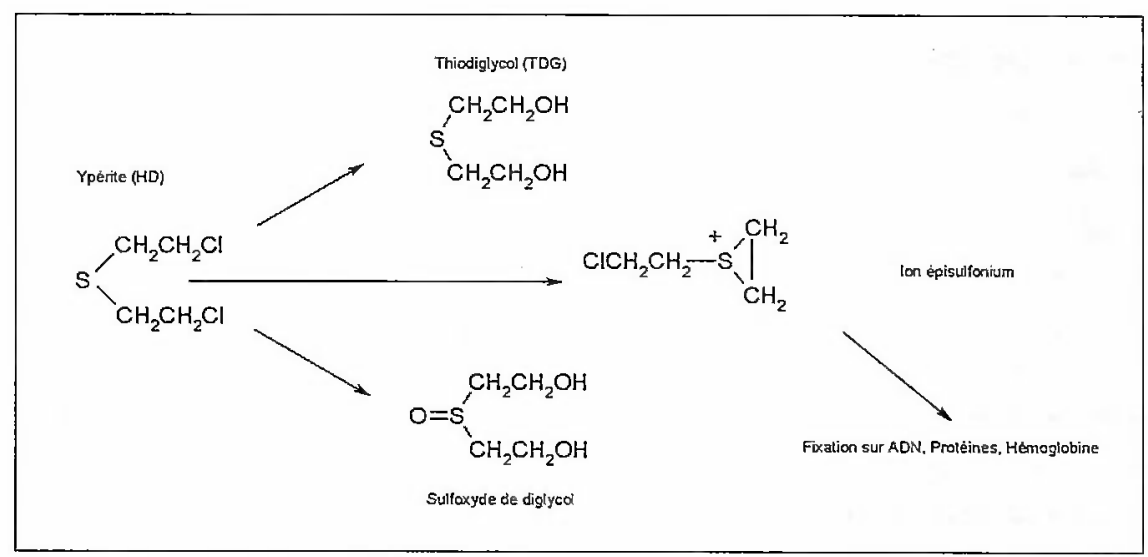

Figure 1: Métabolites de l'ypérite résultant de l'hydrolyse et de l'oxydation.

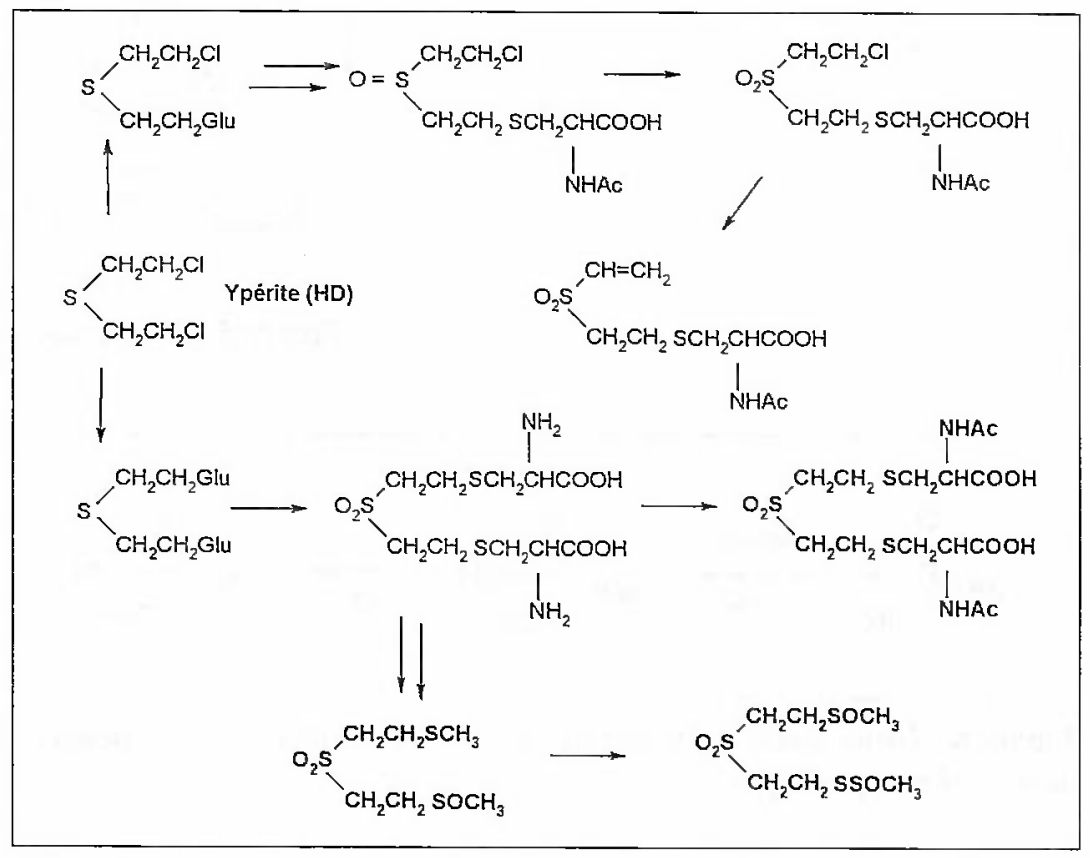

Figure 2 : Formation des métabolites mercapturiques et issus des béta lyases de l'Ypérite après conjugaison au glutathion.

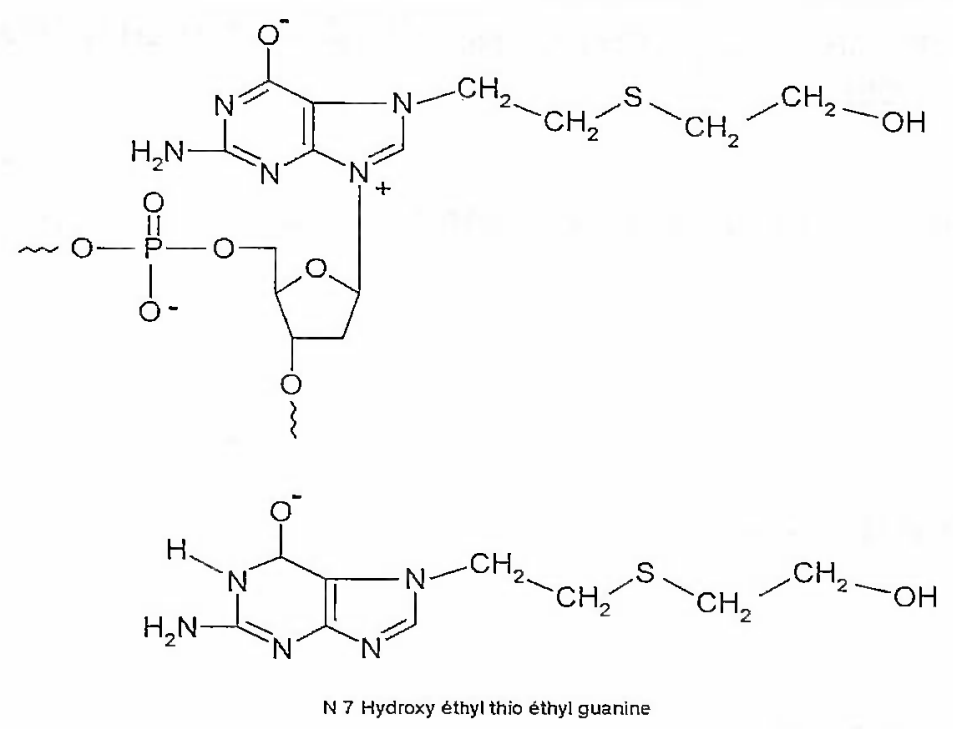

Figure 3 : Formation de l'adduit de l'ypérite sur la guanine : N7 HETE guanine. 
Annales de Toxicologie Analytique, vol. XIX, n² 2, 2007

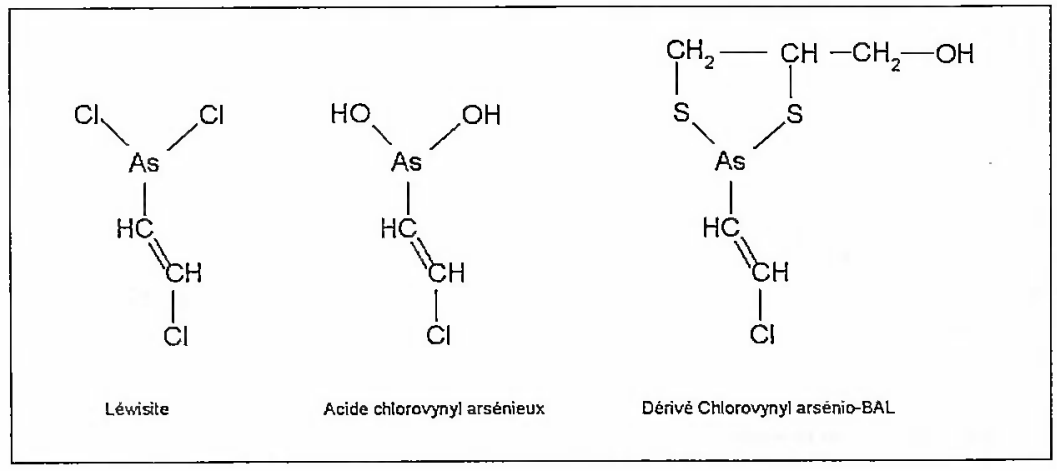

Figure 4 : Léwisite, métabolite issu de l'hydrolyse et produit de dérivation avec le BAL (dimercaptopropanol).

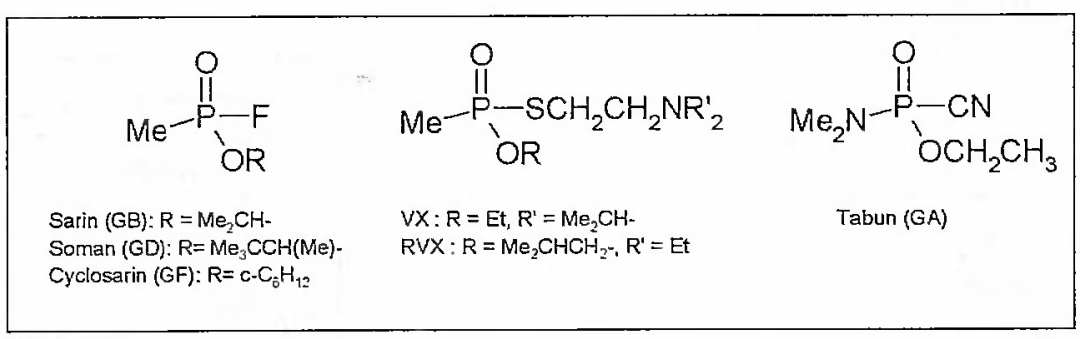

Figure 5 : Principaux neurotoxiques de guerre organophosphorés.

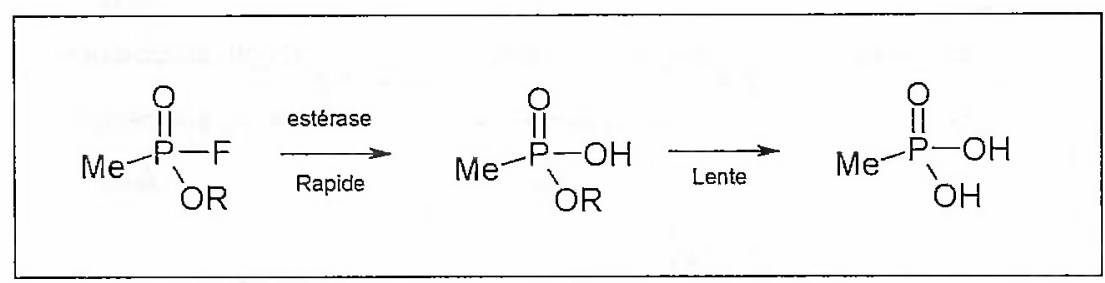

Figure 6 : Formation d'acide méthyl phosphonique alkyl et de très peu d'acide méthyl phosphonique.

$$
\mathrm{VX} \stackrel{\text { estérase }}{\longrightarrow} \mathrm{Me} \stackrel{\stackrel{\mathrm{O}}{\mathrm{P}}-\mathrm{OH}}{\mathrm{O}} \mathrm{OH}+\mathrm{HSCH}_{2} \mathrm{CH}_{2} \mathrm{~N}\left(\mathrm{CHMe}_{2}\right)_{2} \stackrel{\substack{\text { thiol S méthyl } \\ \text { transérase }}}{\longrightarrow} \mathrm{MeSCH}_{2} \mathrm{CH}_{2} \mathrm{~N}\left(\mathrm{CHMe}_{2}\right)_{2}
$$

Figure 7 : Formation d'éthyl méthyl phosphonate (EMPA) et de méthyl thio éthyl diisopropylamine.

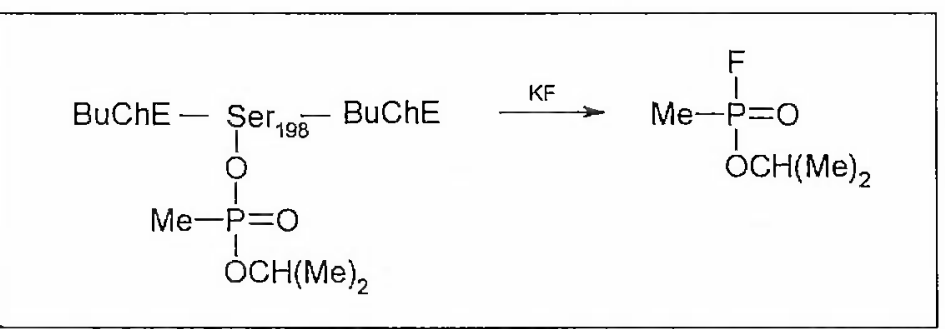

Figure 8 : Réactivation de la butyrylcholinestérase par le fluorure de potassium ; libération du sarin. 


\section{Références}

1. Ricordel I., Meunier J., Armes chimiques : Antidotes. Aperçu sur les moyens actuels, perspectives. Ann Pharm Fr. $2000: 58: 5-12$.

2. Lallement G., Utilisation d'un « Gaz » dans le Théâtre de Moscou : données toxicologiques. Médecine et armées. 2004 ; 32, 2 : 109-111.

3. Ricordel I., Renaudeau C., Pailler F.M., Noto R., Blanchet J.M. Les agressifs chimiques. France Sélection. Paris. $1997: 75-117$

4. Lacoste A., Gastaldon L., Biot F., Pons D., Ledreff P. Identification des agents chiniques à la suite d'un attentat. Urgence pratique $2004 ; 66: 29-35$.

5. Ricordel I., Renaudeau C., Bellier B., Coppet L. Analyses de contrôle de la contamination chimique. Techniques de l'ingénieur. Traité Analyse et Caractérisation. 2002 : P3245 : 1-23.

6. Ricordel I. Toxicologie des agressifs chimiques. In Campana J.P. ed. Principes de la médecine légale. Arnette. $2003: 203-211$.

7. Black R. M., Noort D. Methods for the Retrospective Detection of Exposure to Toxic Scheduled Chemicals. Part A : Analysis of Free Metabolites. In : Mesilaakso M. Chemical Weapons Convention Chemical Analysis. John Wiley and Sons Interscience, Chichester. 2005 Chapter 16 : 419-447.

8. Noort D., Black R. M., Methods for the Retrospective Detection of Exposure to Toxic Scheduled Chemicals. Part B : Mass Spectrometric and Immunochemical Analysis of Covalent Adducts to Proteins and DNA. In Mesilaakso M. Chemical Weapons Convention Chemical Analysis. John Wiley and Sons Interscience, Chichester. 2005 Chapter $17: 448-474$.

9. Noort D., Benschop H.P., Black R.M., Biomonitoring of Exposure to Chemical Warfare Agents: A review. Toxicol. Appl. Pharmacol. 2002 ; 184 : 116-126.

10. Maisonneuve A., Callebat I., Desbordes L., Coppet L. Specific and Sensitive Quantification of 2,2'Dichloroethyl Sulfide, Sulphur Mustard, in Water, Plasma and Blood Application to its Kinetic Study in the Rat after Intravenous Intoxication. J. Chomatogr. B. 1992 ; 583 : $155-165$

11. Black R. M., Read R.W., Improved Methodology for Detection and Quantification of Urinary Metabolites of Sulphur Mustard Using Chromatography-Tandem Mass Spectrometry. J. Chromatogr. B. $1995: 665: 97-105$.

12. Black R. M., Read R.W. Methods for Analysis of Thiodiglycol. Sulphoxide, a Metabolite of Sulphur Mustard, in Urine Using Gas Chromatography-Mass Spectrometry. J. Chromatogr. $1991 ; 558$ : 393-404.

13. Fidder A., Noort D., De Jong LPA., Benschop H.P., Hults A.G. N7-(2-Hydroxyethylthioethyl)-guanine : a Novel Urinary Métabolite Following Exposure to Sulphur Mustard. Arch. Toxicol. $1996 ; 70: 854-855$.

14. Ludlum D. B., Austin-Ritchie P., Hagopian M., Niu T-Q, Yu D. Detection of Sulfur Mustard-Induced DNA Modification. Chem.-Biol. Interact. 1994 ; 91 : 39-49.
15. Black R. M., Clarke R.J., Harrison J.M., Read R.W. Biological Fate of Sulphur Mustard: Identification of Valine and Histidine Adducts of Haemoglobin from Casualties of Sulphur Mustards Poisoning. Xenobiotica. $1997 ; 27: 499-512$.

16.Noort D., Hults A.G., Trap H.C., De Jong L.P.A., Benschop H.P. Synthesis and Mass Spectrometric Identification of the Major Amino Acid Adduct Formed Between Sulphur Mustard and Haemoglobin in Human Blood. Arch. Toxicol. 1997 ; 71 : 171-178.

17. Price E.O., Smith J.R., Clark C.R., Schlager J.J., Shih M.L. MALDI TOF/MS as a Diagnostic Tool for the Confirmation of Sulfur Mustard Exposure. J. Appl. Toxicol. 2002; 20: S193-S197.

18. Noort D., Hults A.G., De Jong L.P.A., Benschop H.P. Alkylation of Human Serum Albumin by Sulfur Mustard In Vitro and In Vivo: Mass Spectrometric Analysis of a Cystein Adduct as a Sensitive Biomarker of Exposure. Chem. Res. Toxicol. $1999 ; 12: 715-721$.

19. Korte W. D., Smith J.R., Capacio B.R., Delion M., Andersen D.R. Monitoring Sulfur Mustard Exposure by GC-MS Analysis of Thiodiglycol Cleaved from Blood Proteins. Army Medical Research and Material Command, Proceedings of the Bioscience Review, 2002 : 269.

20. Van der Schans GP, Noort D., Mars-Groenendijk RH., Fidder A., Chau LF., de Jong LP., Benschop HP. Immunochemical detection of sulfur mustard adducts with keratins in stratum corneum of human skin. Chem Res. Toxicol. 2002;15, 1: 21-25.

21.Lemire S. W., Ashley D.L., Calafat A.M. Quantitative Determination of the Hydrolysis Products of Nitrogen Mustards in Human Urine by Liquid ChromatographyElectrospray Ionization Tandem Mass Spectrometry. J. Anal. Toxicol. $2003 ; 27: 1-6$.

22. Jacubowski E.M., Snith J.R., Logan T.P., Wiltshire N.D., Woodard C.L., Evans R.A., Dolzine T.W. Verification of Lewisite Exposure. Defense Bioscience Revew, US Army Medical Research of Chemical Defense. APG, MD, 10-13 May 1993: 361-368.

23. Fidder A., Noort D., Hulst A.G., De Jong L.P.A., Benschop H.P. Biomonitoring of Exposure to Lewisite Based on Aducts to Haemoglobin. Arch. Toxicol. 2000; $74: 207-214$

24. Wooten J. V., Aschley D.L., Calafat A.M., Quantification of 2-chlorovinylarsenous Acid in Human Urin by Automated Solid-Phase Microextraction-Gas Chromatography-Mass Spectrometry. J. Chromatogr. B. $2002 ; 772$ : 147-153.

25. Fredriksson S.-A., Hammarström L.G., Henriksson L., Lakso H.-A. Trace Determination of Alkyl Methylphosphonic Acids in Environmental and Biological Samples using Gas Chromatography/negative-ion chemical ionization Mass Spectrometry and Tandem Mass Spectrometry. J. Mass Spectrom. 1995 ; 30 : 1133-1143.

26. Miki A., Katagi M., Tsuchihashi H., Yamashita M. Determination of Alkylmethylphosphonic Acids, the Main Metabolites of Organophosphorus Nerve Agents, in Fluids 
by Gas Chromatography Mass Spectrometry by Liquid-Liquid-Solide-Phase Transfer-Catalytized Pentafluorobenzylation. J. Anal. Toxicol. 1999 ; 23 : 8393.

27. Noort M D., Hulst A.G., Platenburg D.H.J.M., Polhuijs M., Benschop H.P. Quantitative Analysis of O-isopropyl Methylphosphonic Acid in Serum Samples of Japanese Citizens Allegedly Exposed to Sarin : Estimation of Internal Dosage. Arch. Toxicol. 1998 ; 72 : 671-675.

28. Polhuijs M.. Langeberg J.P., Benschop H.P. New Method for Retrospective Detection of Exposure to Organophosphorus Anticholinesterases : Application to alleged Sarin Victims of Japanese Terrorists. Toxicol. Appl. Pharmacol. 1997 ; $146: 156-161$.

29. Jakubowski E. M., Heykamp L.S., Durst H.D., Thomson S.A. Preliminary Studies in the Formation of Ethyl Methyl Phosphonofluoridate from Rat and Human Serum Exposed to VX and Treated with Fluorid Ion. Anal. Lett. $2001 ; 34: 727-737$.

30. Nagao M., Takatori T., Matsuda Y., Nakajima M., Iwase H., Iwadate K. Definitive Evidence for the Acute Sarin Poisoning Diagnosis in The Tokyo Subway. Toxical. Appl. Pharmacol. 1997 ; 144 : 198-203.

31. Doom J.A., Schall M., Gage D.A., Talley T.T., Thompson C.M., Richardson R.J. Identification of Butiryl Cholinesterase Adducts after inhibition with Isomalathion using Mass Spectrometry : Difference in Mechanism Between (1R) and (1S) Stereoisomers. Toxicol. Apply. Pharmacol. $2001 ; 173$ : 73-80.

32. Sun J., Lynn B.C. Development of Maldi-TOF-MS Method to Identify and Quantify Butirylcholinesterase Inhibition Resulting from Exposure to Organophosphate and Carbamate Pesticides. American Society for Mass Spectrometry. 2007 ; 1044-0305 (à paraître)

33. Fidder A., Noort D., Hulst A.G., De Ruiter R., Van Der Schans M.J., Benschop H.P. Langerberg J.P. Retrospective Detection of Exposure to Organophosporus Anticholinesterases : Mass Spectrometric Analysis of Phosphylated Human Butirylcholisterases. Chem. Res. Toxicol. $2002 ; 15: 582-590$.

34. Black R.M., Harrison J.M., Read R.W. The Interaction of Sarin and Soman with Plasma Proteins : the Identification of a Novel Phosphorylation Site. Arch. Toxicol. 1999 ; $73: 123-126$.

35. Elhanany E., Ordenlich A., Dgany O., Kaplan D., Segall Y., Barak R., Velan B., Shafferman A. Resolving Pathways of Interaction of Covalent Inhibitors with the Activ Site of Acetylcholinesterases : MALDI-TOF/MS Analysis of various Nerve Agent Phosphyl Adducts. Chem res. Toxicol. $2001 ; 14: 912-918$.

36. Li B., Schopfer L.M., Hinrichs S.H., Masson P., Lockridge O. Matrix-assisted desorption/ionization Time-of-Flight Mass Spectrometry Assay for Organophosphorus Toxicants Bound to Albumin at Tyr411. Analytical Biochemistry. 2007; 361: 263-272.

37. Peeples E.S., Schopfer L.M., Duyssen E.G., Spaulding R., Voelker T., Thompson C.M., Lockridge O. Albumin, a New Biomarker of Organophosphorus Toxicant Exposure Identified by Mass Spectrometry. Toxicological Sciences. $2005: 83: 303-312$.
38. Lallement G., Clair P., Zabe D.; A new auto-injector for emergency self-treatment of nerve gas poisoning with an improved pharmaceutical concept and design, Applied science and Analysis. 2007; $118: 24-25$.

39. Dorandeu F., Mikler J., Thiermann H., Tenn C., Davidson C., Sawyer T., Lallement G., Worek F. Swine models in the design of more effective medical countermeasures against organophosphorus poisoning. Toxicology. 2007 ; 233 (1) : 128-144.

40. Lundy P.M., Raveh L., Amitai G., Development of the bisquaternary oxime HI-6 toward clinical use in the treatment of organophosphate nerve agent poisoning. Toxicology Review. 2006 ; 25(4): 231-243.

41. Dorandeu F., Baille V., Mikler J., Testylier G., Lallement G., Sawyer T., Carpentier P. Protective effects of $\mathrm{S}(+)$ ketamine and atropine against lethality and brain damage during soman-induced status epilepticus in guinea-pigs. Toxicology. 2007; 234(3) : 185-193.

42. Shih T.M., Rowland TC., McDonough JH. Anticonvulsants for nerve agent-induced seizures: the influence of the therapeutic dose of atropine. J. Pharmacology and Experimental Therapeutics. 2007 ; 320(1) : 154-161.

43. Lallement G., Clarençon D., Galonnier M., Baubichon D.. Burkart M.F. Acute Soman Poisoning Primates Neither Pretreated nor Received Immediate Therapy: value of Gacyclidine (GK 11) in delayed medical support. Arch. Toxicol. $1999 ; 73: 115-122$.

44. Lallement G., Veyret J., Masquelez C., Aubriot S., Burkart M.F., Baubichon D. Efficacy of Huperzine in Preventing Soman Induced Seizures, Neuropathological Changes and Lethality. Fundam. Clin. Pharmacol. 1997 ; 11 : 387-394.

45. Masson P., Nachon F., Rochu D . Ingénerie d'enzymes pour la protection, la décontamination et le traitement des agressions par le composé organophosphorés. Bul. Acad. Natle. Méd. 2007 ; 191 ; 93-112.

46. Poyot T., Nachon F., Rochu D., Fournier D., Masson P. Optimiser les enzymes, mutagénèse et évolution dirigées. Ann. Pharm. Fr. $2007 ; 65: 119-125$.

47. Germain M., Paquereau L., Winterhalter M., Hochepied J.-F., Fournier D. Liposomes: Support à la formation de capsules plus stables à base de polyélectrolytes reticules ou de silice. Ann. Pharm. Fr. 2007 ; 65 : 134-141.

48. Moralès R., Berna A., Carpentier P., Contreras-Martel C., Renault F., Nicodème M., Chesne-Seck M.-L., Bernier F., Dupuy J., Schaeffer C., Diemer H., Van-Dorsselaer A., Fontecilla-Camps J.C., Masson P., Rochu D., Chabrière E.. Découverte et Structure cristallographique d'une apolipoprotéine humaine. Ann. Pharm. Fr. 2007 ; 65 : 98-107.

49. Masurier N., Lafont O., Estour F. Les cyclodextrines substituées : Un exemple de catalyseur biomimétique. Ann. Pharm. Fr. 2007 ; 65 : 126-133.

50. Rice P; Sulphur mustard injuries of the skin. pathophysiology and management, Toxicology Review. 2003 ; 22, 111-118.

51. Filliat P., Coubard S., Pierard C., Liscia P., Beracochea D., Four E., Baubichon D., Masqueliez C., Lallement G., Collombet J-M. Long term behavioral consequences of soman poisoning in mice. Neurotoxicology. 2007; 28 : 508-519. 